SMUHEP/98-04

\title{
The Mass Operator in the Light-Cone Representation
}

\author{
Gary McCartor \\ Department of Physics, Southern Methodist University, Dallas, TX 75275
}

\begin{abstract}
I argue that for the case of fermions with nonzero bare mass there is a term in the matter density operator in the light-cone representation which has been omitted from previous calculations. The new term provides agreement with previous results in the equal-time representation for mass perturbation theory in the massive Schwinger model. For the DLCQ case the physics of the new term can be represented by an effective operator which acts in the DLCQ subspace, but the form of the term might be hard to guess and I do not know how to determine its coefficient from symmetry considerations.
\end{abstract}

\section{INTRODUCTION}

In the light-cone representation, if the Fermi field has nonzero bare mass the field $\psi_{-}$ is usually treated as a pure constraint. That is, the equation of motion for $\psi_{-}$- which contains no $x^{+}$-derivatives - is solved to write $\psi_{-}$in terms of the true degrees of freedom. Solving the equation of motion requires the use of boundary conditions however, and I shall argue in this letter that a careful analysis shows that the boundary conditions which should be chosen require additional degrees of freedom and that these degrees of freedom lead to a term in the mass operator which has been left out of — as far as I am aware — all previous light-cone calculations.

That the $\psi_{-}$field contains degrees of freedom for the case of zero bare mass has been known for some time [0 [3]; the case of free fields is enough to show that. Here I shall argue that the case of nonzero bare mass is not qualitatively different (regarding this point, see also Refs. [4.5]). I shall consider the case of the massive Schwinger model. The existence of an exact solution for the massless case and a large body of results in the equal-time representation for the massive case makes the argument particularly sharp. In the case of the continuum, and with the new term present, simple mass perturbation theory gives results in agreement with mass perturbation theory in the equal-time representation [6 \&] and for surfaces "near" the light-cone [9]. For the case of periodicity conditions on the characteristic (DLCQ) [10,11] there is a disagreement with the older calculations. I shall argue that the noncovariant regulation of the singularity at $p^{+}=0$ leads to a composite operator renormalization. I believe that this last case provides a useful example for those who wish to devise effective theories which act only in the DLCQ subspace [12, 13]. 


\section{THE SCHWINGER MODEL}

The Schwinger model is electrodynamics of massless fermions in $1+1$ dimensions [14]. In light-cone gauge the operator solution is [15:

$$
\begin{gathered}
\psi_{+}=Z_{+} e^{\Lambda_{+}^{(-)}} \sigma_{+} e^{\Lambda_{+}^{(+)}} \\
\Lambda_{+}=-i 2 \sqrt{\pi}\left(\tilde{\eta}\left(x^{+}\right)+\tilde{\Sigma}\left(x^{+}, x^{-}\right)\right) \\
Z_{+}^{2}=\frac{m^{2} e^{\gamma}}{8 \pi \chi} \\
\psi_{-}=Z_{-} e^{\Lambda_{-}^{(-)}} \sigma_{-} e^{\Lambda_{-}^{(+)}} \\
Z_{-}^{2}=\frac{\chi e^{\gamma}}{2 \pi} \\
\Lambda_{-}=-i 2 \sqrt{\pi} \phi\left(x^{+}\right) \\
A^{-}=\frac{1}{m} \partial_{+}(\tilde{\eta}+\tilde{\Sigma}) \\
A^{+}=0
\end{gathered}
$$

Here, $\phi\left(x^{+}\right)$is the left-moving component of a free, massless scalar field while $\tilde{\eta}\left(x^{+}\right)$is the left-moving component of a free, massless ghost field, both regulated in the infrared with a Klaiber [16] regulator of parameter $\chi . \tilde{\Sigma}$ is a free massive psuedoscalar field of mass $e / \sqrt{\pi}$. $\sigma_{+}$and $\sigma_{-}$are spurions. The vacuum must be chosen to be a theta-state, formed as:

$$
|\Omega(\theta)\rangle \equiv \sum_{n=-\infty}^{\infty} e^{i M \theta}|\Omega(M)\rangle \quad ; \quad|\Omega(M)\rangle=\left(\sigma_{+}^{*} \sigma_{-}\right)^{M}|0\rangle
$$

The physical subspace is formed by applying all polynomials in $\tilde{\Sigma}$ to $|\Omega(\theta)\rangle$. A detailed discussion of this solution is given elsewhere 15 but the most important point for the present discussion is (2.4): the field $\psi_{-}$is not zero but is isomorphic to the left moving component of a free, massless Fermi field - it contains degrees of freedom. We also note that:

$$
\langle\Omega(\theta)|\bar{\psi} \psi| \Omega(\theta)\rangle=-\frac{m}{2 \pi} e^{\gamma} \cos \theta
$$

If we now consider the case of imposing periodicity conditions on the characteristics, in particular: $\psi_{+}\left(x^{-}+2 L\right)=-\psi_{+}\left(x^{-}\right) ; \psi_{-}\left(x^{+}+2 L\right)=-\psi_{-}\left(x^{+}\right)$, we find the solution [3]: 


$$
\begin{aligned}
& \psi_{+}=\frac{1}{\sqrt{2 L}} e^{-\lambda_{+}^{*}(x)} \sigma_{+}(x) e^{\lambda_{+}(x)} \\
& \lambda_{+}(x)=-i \sqrt{\frac{\pi}{L}} \sum_{n=1}^{\infty} \frac{1}{\sqrt{p_{-}(n)}} C(n) e^{-i p(n) x} \\
& p_{-}(n)=\frac{n \pi}{L} \quad ; \quad p_{+}(n)=\frac{m^{2} L}{4 n \pi} \\
& \psi_{-}=\frac{1}{\sqrt{2 L}} e^{-\lambda_{D}^{*}\left(x^{+}\right)} \sigma_{-}(x) e^{\lambda_{D}\left(x^{+}\right)} \\
& \lambda_{D}\left(x^{+}\right)=\sum_{n=1}^{\infty} \frac{1}{\sqrt{n}} D(n) e^{-i k_{+}(n) x^{+}} \\
& \sigma_{+}(x)=e^{-i \frac{\sqrt{\pi}}{4 L m}\left(Q_{+}\left(x^{-}-x^{+}\right)\right)} \sigma_{+}(0) e^{-i \frac{\sqrt{\pi}}{4 L m}\left(Q_{+}\left(x^{-}-x^{+}\right)\right)} \\
& \sigma_{-}(x)=e^{-i \frac{\sqrt{\pi}}{4 L m}\left(Q_{-}\left(x^{+}-x^{-}\right)\right)} \sigma_{-}(0) e^{-i \frac{\sqrt{\pi}}{4 L m}\left(Q_{-}\left(x^{+}-x^{-}\right)\right)} \\
& A^{-}=-\frac{i}{\sqrt{L} m} \sum_{n=1}^{\infty} \frac{p^{-}(n)}{\sqrt{p_{-}(n)}}\left(C(n) e^{-i p(n) x}-C^{*}(n) e^{i p(n) x}\right)-\frac{1}{L m^{2}} Q_{+} \\
& A^{+}=-\frac{1}{L m^{2}} Q_{-}
\end{aligned}
$$

Here, the $C$ 's are the fusion operators associated with bosonizing the $\psi_{+}$field and the $D$ 's are the fusion operators associated with bosonizing the $\psi_{-}$field. The vacuum must be chosen to be a theta-state, formed as:

$$
|\Omega(\theta)\rangle \equiv \sum_{n=-\infty}^{\infty} e^{i M \theta}|\Omega(M)\rangle \quad ; \quad|\Omega(M)\rangle=\left(\sigma_{+}^{*} \sigma_{-}\right)^{M}|0\rangle
$$

The physical subspace is formed by applying all polynomials in $C^{*}$ s to $|\Omega(\theta)\rangle$. A detailed discussion of this solution is given elsewhere [3], but the most important point for the present discussion is (2.14): the field $\psi_{-}$is not zero; it is not quite isomorphic to a free, massless Fermi field due to the fact that the $\sigma_{-}$operator picks up a dependence on $x^{-}$, but the degrees of freedom are the same as for the left moving component of a free, massless Fermi field. We also note that:

$$
\langle\Omega(\theta)|\bar{\psi} \psi| \Omega(\theta)\rangle=-\frac{1}{L} \cos \theta
$$

The chiral condensate goes to zero as the periodicity length, $L$, goes to infinity, which is not the result in the continuum solution presented above [17]. 


\section{THE MASSIVE SCHWINGER MODEL}

Now let us consider adding a mass term, that is, we take the Lagrangian to be:

$$
\mathcal{L}=\frac{1}{2}\left(i \bar{\psi} \gamma^{\mu} \partial_{\mu} \psi-i \partial_{\mu} \bar{\psi} \gamma^{\mu} \psi\right)-\frac{1}{4} F^{\mu \nu} F_{\mu \nu}-A^{\mu} J^{\prime}{ }_{\mu}-\mu \bar{\psi} \psi
$$

The equations of motion are:

$$
\begin{gathered}
\frac{\partial \psi_{+}}{\partial x^{+}}+i \frac{1}{4} e\left(A^{-} \psi_{+}+\psi_{+} A^{-}\right)+i \frac{1}{2} \mu \psi_{-}=0 \\
\frac{\partial \psi_{-}}{\partial x^{-}}+i \frac{1}{4} e\left(A^{+} \psi_{-}+\psi_{-} A^{+}\right)+i \frac{1}{2} \mu \psi_{+}=0 \\
\frac{\partial^{2} A^{-}}{\partial x^{-2}}=-\frac{1}{2} J^{+} \\
-\frac{\partial^{2} A^{+}}{\partial x^{+2}}+\frac{\partial^{2} A^{-}}{\partial x^{+} \partial x^{-}}=\frac{1}{2} J^{-}
\end{gathered}
$$

The equation for $\psi_{-}$, (3.3), contains no $x^{+}$derivatives. If we take the continuum case, where $A^{+}=0$ then we may take:

$$
\psi_{-}=-\int i \frac{1}{2} \mu \psi_{+} d x^{-}
$$

What has usually been done in the past (always, as far as I know) is to define the antiderivative to be such that if:

$$
\psi_{+}\left(x^{-}\right)=\frac{1}{\sqrt{2 L}} \sum_{n=1}^{\infty} b(n) e^{-i k_{-}(n) x^{-}}+d^{*}(n) e^{i k_{-}(n) x^{-}}
$$

then:

$$
\int \psi_{+}\left(x^{-}\right)=\frac{1}{\sqrt{2 L}} \sum_{n=1}^{\infty} \frac{1}{-i k_{-}(n)} b(n) e^{-i k_{-}(n) x^{-}}+\frac{1}{i k_{-}(n)} d^{*}(n) e^{i k_{-}(n) x^{-}}
$$

From now on we shall understand the antiderivative to mean that particular one. But we can also take:

$$
\psi_{-}=\psi_{-}^{0}-\int i \frac{1}{2} \mu \psi_{+} d x^{-}
$$

Where $\psi_{-}^{0}$ is any solution to the homogeneous equation, which in this case means any function of $x^{+}$. Looking at (2.4) we see that if the solution for nonzero $\mu$ is to go smoothly into the Schwinger model we should not take $\psi_{-}^{0}$ to be zero but rather:

$$
\psi_{-}^{0}=Z_{-} e^{\Lambda_{-}^{(-)}} \sigma_{-} e^{\Lambda_{-}^{(+)}}
$$


With that choice we find that in the physical subspace the operator $P^{-}$gets a correction

$$
\begin{aligned}
\delta P^{-}=\mu Z_{-} & Z_{+} \int_{-\infty}^{\infty}\left(\sigma_{-}^{*} \sigma_{+}: e^{-i \sqrt{\pi} \tilde{\Sigma}\left(0, x^{-}\right)}:+C . C .\right) d x^{-} \\
& +\frac{1}{2} \mu^{2} \int_{-\infty}^{\infty}\left(\psi_{+}^{*}\left(0, x^{-}\right)\left[-\int i \frac{1}{2} \psi_{+}\right]+C . C .\right) d x^{-}
\end{aligned}
$$

The first term depends on the vacuum angle, $\theta$, while the second term does not. Furthermore, the second term needs regularization - a very important issue, but one that need not concern us here. One might expect a factor of one-half in front of the first term, but the equation is correct as it stands as can be verifyed by checking the Heisenberg equation for the $x^{+}$ derivative of $\psi_{+}$. The constants $Z_{-}$and $Z_{+}$are wavefunction renormalization constants; they depend on $\mu$ and are determined by the requirement that the fields remain canonically normalized. We know from the Schwinger model solution that:

$$
\begin{aligned}
& Z_{-}=Z_{+}(\mu)=\sqrt{\frac{\chi e^{\gamma}}{2 \pi}}+\mathcal{O}(\mu) \\
& Z_{+}=Z_{+}(\mu)=\sqrt{\frac{m^{2} e^{\gamma}}{8 \pi \chi}}+\mathcal{O}(\mu)
\end{aligned}
$$

If we perform a straightforward first order perturbation calculation for the state of one Schwinger particle:

$$
|p\rangle \equiv \tilde{\Sigma}^{*}(p)|\Omega(\theta)\rangle
$$

only the first term contributes and we get:

$$
\delta M^{2}=\left\langle p\left|P^{+} \delta P^{-}\right| p\right\rangle=2 m \mu e^{\gamma} \cos \theta
$$

This result is in agreement with previous calculations done in the equal-time representation, both in the continuum [6,7] and with periodicity conditions [8,18], and in a representation using a periodic surface "near" the light-cone [9]. Indeed, for the case of the equal-time, continuum calculations, it is easy to evaluate the fields on the characteristics (to first order in $\mu$ ) and see that, not only are the results numerically equal, but the operators are the same and so the calculations are completely equivalent [19].

If we now turn to the DLCQ case, by the same argument we would choose:

$$
\psi_{-}^{0}=\frac{1}{\sqrt{2 L}} e^{-\lambda_{D}^{*}\left(x^{+}\right)} \sigma_{-}(x) e^{\lambda_{D}\left(x^{+}\right)}
$$

That will lead to a new term in $P^{-}$operator which, in the physical subspace is given by:

$$
\begin{gathered}
\delta P^{-} \subset \mu \sigma_{-}^{*} \sigma_{+} \int_{-L}^{L} \frac{1}{2 L} e^{\lambda_{+}^{(-)}\left(0, x^{-}\right)} e^{\lambda_{+}^{(+)}\left(0, x^{-}\right)} d x^{-}+C . C . \\
\lambda_{+}(x)=-i \sqrt{\frac{\pi}{L}} \sum_{n=1}^{\infty} \frac{1}{\sqrt{p_{-}(n)}}\left(C(n) e^{-i p(n) x}+C^{*}(n) e^{i p(n) x}\right)
\end{gathered}
$$


Note that we may replace the operator $\sigma_{-}^{*} \sigma_{+}$by $e^{i \theta}$ and similarly for the complex conjugate, and thus have an operator which operates entirely within the DLCQ subspace - an important point from the point of view of effective theories.

The state of one Schwinger particle is:

$$
|n\rangle \equiv C^{*}(n)|\Omega(\theta)\rangle
$$

And mass perturbation theory gives:

$$
\left\langle n\left|P^{+} \delta P^{-}\right| n\right\rangle=4 \pi \mu \frac{1}{L} \cos \theta
$$

The mass shift goes to zero as $L$ goes to infinity for the same reason that the chiral condensate goes to zero as $L$ goes to infinity.

Introducing periodicity conditions on $x^{+}=0$ regulates the singularity at $p^{+}=0$ in a very abrupt way which violates the Poincaré symmetries, particularly parity. Thus we should not expect to absorb all the infinities into mass, wavefunction and coupling renormalizations. The operator mixes with itself under renormalization and we should write:

$$
\delta P^{-} \subset Z \mu \sigma_{-}^{*} \sigma_{+} \int_{-L}^{L} \frac{1}{2 L} e^{\lambda_{+}^{(-)}\left(0, x^{-}\right)} e^{\lambda_{+}^{(+)}\left(0, x^{-}\right)} d x^{-}+C . C .
$$

where $Z$ is not determined by requiring canonical normalization of the fields but must be determined by fitting to data. Taking the continuum calculation as data we write:

$$
Z=Z(\mu)=\frac{L m e^{\gamma}}{2 \pi}+\mathcal{O}(\mu)
$$

While this should perhaps be regarded as a conjecture at the moment, it is testable within the context of the model: having chosen $Z$ to fit the mass we can then unambiguously predict the S-matrix. Since the massive Schwinger model has a nontrivial S-matrix, we have a nontrivial test of the conjecture. I hope to report results on that test in the near future.

\section{EFFECTIVE THEORIES}

I believe the example we have been considering contains at least three important lessons for those who wish to make effective theories which act in the DLCQ subspace and incorporate the effects of zero modes with additional operators [12,13]. The first is good news: the effects of zero modes we have been considering here can in fact be incorporated into effective operators. But the second is that the form of the operators may be hard to guess, particularly if one thinks entirely within the DLCQ framework. Since the $\psi_{+}$field is irreducible in the DLCQ subspace we are guaranteed to be able to write our effective operator in terms of it; indeed we can and it seems rather natural if we bosonize the field and think of zero modes. As a functional of $\psi_{+}$it is:

$$
\begin{aligned}
Z \mu e^{-i \theta} \frac{1}{2 L} \int_{-L}^{L} d x^{-}: \lim _{y^{-} \rightarrow 0} e^{\left(2 i \pi \int\left[: \psi_{+}^{*}\left(y^{-}\right) \psi_{+}\left(y^{-}\right):-\frac{1}{2 L} \int_{-L}^{L}: \psi_{+}^{*}\left(z^{-}\right) \psi_{+}\left(z^{-}\right): d z^{-}\right]\right)^{(-)}} \psi_{+}\left(y^{-}\right) \\
\times e^{\left(2 i \pi \int\left[: \psi_{+}^{*}\left(y^{-}\right) \psi_{+}\left(y^{-}\right):-\frac{1}{2 L} \int_{-L}^{L}: \psi_{+}^{*}\left(z^{-}\right) \psi_{+}\left(z^{-}\right): d z^{-}\right]\right)^{(+)}} \psi_{+}^{*}\left(x^{-}\right):+C . C .
\end{aligned}
$$


While this operator can certainly be found I think it may not be the first thing that would pop into everyone's head.

The final point is that even if the form of the operator is given, the coefficient cannot always be found by restoring the broken symmetry. Here the relevant symmetry is parity but the effective operator does not restore parity. One can, in fact, find a similar operator which does restore parity (and gives the correct limit of the chiral condensate) but it does not act in the DLCQ subspace [2]. It requires a great enlargement of the DLCQ space in a way that breaks the periodicity conditions. I believe that the operator we have been studying here has an analog in four dimensions and that the analog operator probably plays

a role in chiral symmetry breaking. I do not yet know what the form of the operator is and I expect that the coefficient will have to be fit to data.

\section{ACKNOWLEDGMENTS}

I appreciate the efforts of Dave Robertson and Eliana Vianello who helped me understand the factors in (3.11). This work was supported in part by grants from the U.S. Department of Energy. 


\section{REFERENCES}

[1] G. McCartor, Z. Phys. C 41, 271 (1988).

[2] G. McCartor, Z. Phys. C 52, 611 (1991).

[3] G. McCartor, Z. Phys. C 64, 349 (1994).

[4] G. McCartor, Talk presented at the Iowa State meeting Ames Iowa, June, 1996.

[5] D. G. Robertson, Talk presented at Orbis Scientiae, Miami Beach, FL., Jan. 25-28, 1996; hep-th/9603205.

[6] S. Coleman, R. Jackiw and L. Susskind, Ann. Phys. (N.Y.) 93, 267 (1975).

[7] S. Coleman, Ann. Phys. (N.Y.) 101, 239 (1976).

[8] T. Banks, J. Kogut and L. Susskind, Phys. Rev. D 13, 1042 (1976).

[9] T. Fields, H.-J. Pirner and J. Vary, Phys. Rev. D 53, 2001 (1996).

[10] T. Maskawa and K. Yamawaki, Prog. Theor. Phys. 56, 270 (1976).

[11] H.-C. Pauli and S. J. Brodsky, Phys. Rev. D 32, 7231 (1985).

[12] K. G. Wilson and D. G. Robertson, in Theory of Hadrons and Light-Front QCD, Stanislaw D Głazek, ed. (World Scientific, 1995); hep-th/9411007.

[13] M. Burkardt, hep-ph/9611416

[14] J. Schwinger, Phys. Rev. 128, 2425 (1962).

[15] Y. Nakawaki and G. McCartor, in preparation.

[16] B. Klaiber, Boulder Lectures in Theoretical Physics (Gordon and Breach,1967), vol.XA.

[17] G. McCartor, Int. J. Mod. Phys. A 12, 1091 (1997).

[18] Y. Nakawaki, Prog. Theor. Phys. 70, 1105 (1983).

[19] G. McCartor, D. G. Robertson and E. Vianello, in preparation. 